\title{
Neuroscience
}

\section{Brain's immune cells put the brakes on neurons}

\section{Thomas Pfeiffer \& David Attwell}

\section{Microglia are the brain's immune cells. A previously unknown role for microglia has now been uncovered - providing negative feedback to active neurons to help the brain process information. See $\mathbf{p .} X X X$.}

Neural circuits in the brain rely on neuronal excitation (a positive change in the electrical potential across the cell membrane), combined with delayed inhibition (Fig. 1a, b). Inhibition is crucial for keeping neuronal activity in the optimal range for encoding information, minimising brain energy use and computing useful neuronal outputs. It has classically been thought that inhibition is mediated by 'interneurons' that release neurotransmitter molecules (such as the amino acid GABA) to make the membrane potential of the downstream neuron more negative - although neurotransmitter release from nonneuronal cells called astrocytes can also contribute ${ }^{1}$. Writing in Nature, Badimon et al. ${ }^{2}$ extend this repertoire of inhibitory influences to include microglia, the resident immune cells of the brain. Their work raises fascinating questions about the role of microglia in information processing.

Badimon and colleagues took advantage of the fact that blocking activation of the growth-factor receptor protein CSF1R in mice leads to a lack of microglia ${ }^{3}$. The authors found that, if they gave neurostimulants to animals that lacked microglia, the drugs produced long-lasting epileptic seizures, indicative of hyperactive neuronal excitation. Seizures were not observed in wild-type animals receiving the same drugs, indicating that microglia normally exert a brake on neuronal activity. This result echoes and extends two previous studies $^{4,5}$. Microglial processes are attracted to the cell bodies of active neurons by the release of ATP, where they decrease neuronal activity both in normal ${ }^{4}$ and in pathological ${ }^{5}$ conditions.

Whereas these previous studies focussed on cell bodies, Badimon and colleagues focussed on the synaptic junctions between neurons, which also release ATP to attract microglial processes. ATP is converted by the microglial enzyme CD39 into ADP (and then to AMP). ADP activates $P 2 Y_{12}$ receptor proteins found only on microglia ${ }^{6,7}$. Blocking $P 2 Y_{12}$ receptors has been shown to inhibit the attraction of microglia to cell bodies and synapses ${ }^{5}$. Badimon et al. found that $\mathrm{P}_{2} \mathrm{Y}_{12}$ blockade also reduces neuronal inhibition by microglia in response to neurostimulants. 
How might microglia-neuron interactions inhibit the electrical activity of neurons? The authors found that deleting microglia decreased extracellular levels of the molecule adenosine (ADO). Pharmacologically blocking CD39 or the downstream enzyme CD73 (which converts AMP into ADO) also lowered ADO levels. Furthermore, blocking the activity of CD39 increased the susceptibility of mice to seizures in response to neurostimulants. Together, these observations implicate ADO as the microglial-derived factor that dampens neuronal activity.

ADO is well-known to lower neuronal excitability ${ }^{8}$. Indeed, the reason that coffee makes us more alert is that caffeine blocks ADO's inhibitory effects. ADO lowers excitability by acting on its $A 1$ receptors, which (by lowering the concentration of the intracellular messenger cyclic AMP) decrease the release of the excitatory neurotransmitter glutamate and reduce its postsynaptic effects. A1 receptors also activate potassium ion channels in neuronal membranes to keep their membrane potential negative (and so keep the neurons unexcited). Thus, Badimon et al. and colleagues have uncovered a previously unknown feedback loop for neuronal regulation mediated by microglia, which, when attracted to active synapses, generate ADO to inhibit excessive neuronal activity (Fig. 1c).

The authors showed that this negative feedback operates in a region-specific manner. Deletion of microglia in the brain's grey matter (where neurons have their cell bodies and synapses) caused the hyperactive neuronal response to mild excitation. By contrast, deleting microglia in white matter (where long-range neuronal connections run) did not cause hyperactivity. In addition, deletion of microglia in specific regions of grey matter affected only those regions, rather than causing excessive activity across the whole brain.

Just how spatially- and temporally-specific might the feedback mechanism be? Two factors should slow its activity. First, there will be a lag between release of ATP by a synapse and the production of local ADO after microglial processes are drawn to that synapse. Second, it is unclear whether the enzymes CD39 and CD73 are close enough spatially for rapid ADO production. Although microglia express CD39 highly, they only weakly express CD73, which is expressed more in other brain cells, such as neurons and cells of the oligodendrocyte lineage ${ }^{6,7}$ (Fig. 1c). Another enzyme (tissue nonspecific alkaline phosphatase, TNAP) can also convert $\mathrm{AMP}$ to $\mathrm{ADO}^{9}$, but this is largely expressed in astrocytes $^{6,7}$. Thus, after microglial CD39 has converted ATP to ADP and AMP, the AMP molecule might have to diffuse some distance, to a different cell type, to be converted to $A D O$. This would lengthen the time course of the feedback loop compared to conventional GABA-mediated synaptic inhibition, which operates within about 50 milliseconds of neuron stimulation (Fig. 1b). The ADO feedback loop might have longer lasting effects, and also be less spatially specific - whereas synaptic inhibition involves direct contacts with target 
neurons, diffusion of ADO precursors implies that the microglial mechanism would act on multiple neurons in an area.

Consistent inhibition of neuronal synapses can cause a decrease in the strength of the connection between neurons. Synapses that are weakened in this way are sometimes removed by microglia or astrocytes in a process called pruning ${ }^{10}$. It will be interesting to determine whether ADO-mediated weakening of synapses triggers this pruning mechanism.

Another question is to what extent the inhibitory influence of microglia depends on the amount of neuronal excitation occurring. Badimon et al. used neurostimulants that affect many neurons. But it remains to be seen whether ADO-mediated inhibition also operates (to a lesser extent) when there is a small amount of excitation. In other words, is this system an emergency brake for extreme situations, or does it act proportionally for all levels of excitation? Inhibitory interneurons have increased influence as neuronal excitation increases - this enables neural circuits to respond differentially to a broader range of input strengths ${ }^{11}$. Microglia-facilitated ADO production might similarly enhance the coding range of neural circuits.

ADO derived from ATP released by astrocytes is proposed to regulate sleep onset ${ }^{12}$. Badimon et al. found that the extracellular level of ADO was reduced by $85 \%$ in a brain region called the striatum in anaesthetised mice lacking microglia, compared to in control mice that had microglia. This suggests that the build-up of extracellular ADO that generates sleep pressure might largely be derived from the activity of microglial CD39. Thus, microgliafacilitated negative-feedback control of neuronal activity could be a side effect of the evolution of a system to induce sleep (or vice versa).

There are also hints that this feedback system might contribute to neurological or psychiatric disease. As Badimon and colleagues show, epileptic seizures can result if microglial-mediated negative feedback is absent. In less-extreme situations, both $\mathrm{P}_{2} \mathrm{Y}_{12}$ receptors and CD39 are downregulated in a range of diseases in which the immune-defence role of microglia is activated, including Alzheimer's disease, Huntington's disease and injection of the bacterial coat protein lipopolysaccharide to mimic bacterial infection, as summarized in Extended Data Fig. 10 of the paper. All of these conditions can also involve increases in neuronal activity. Conversely, upregulation of CD39 can lead to depression-like behaviour ${ }^{13}$.

Going forward, it will be crucial to define the mechanisms of ATP release from neurons, and the spatial and temporal scales on which ADO acts. It also remains to be seen whether there is any role for ADO's lower-affinity A2 receptors in microglial-mediated neuronal inhibition. Finally, do circadian rhythm and disease-related factors modulate these mechanisms? How these immune cells regulate information processing is just beginning to be unravelled. 
Thomas Pfeiffer and David Attwell are in the Department of Neuroscience, Physiology \& Pharmacology, University College London, London WC1E 6BT, UK.

e-mail: d.attwell@ucl.ac.uk

1. Araque, A. et al. Neuron 81, 728-739 (2014). doi: 10.1016/j.neuron.2014.02.007.

2. Badimon, A. et al. Nature XXX, P1-P2 (2020). doi: XXXXX

3. Ginhoux, F. et al. Science 330, 841-845 (2010). doi: 10.1126/science.1194637

4. Li, Y., Du, X.F., Liu, C.S., Wen, Z.L. \& Du, J.L. Dev. Cell 23, 1189-1202 (2012) doi: 10.1016/j.devcel.2012.10.027.

5. Cserép, C., et al. Science 367, 528-537 (2020) doi: 10.1126/science.aax6752.

6. www.brainrnaseq.org

7. www.mousebrain.org/genesearch

8. Haas, H.L. \& Selbach, O. Naunyn Schmiedeberg's Arch. Pharmacol. 362, 375-381 (2000). doi: $10.1007 / \mathrm{s} 002100000314$.

9. Zhang, D., et al. PLoS One 7, e39772 (2012). doi: 10.1371/journal.pone.0039772.

10. Chung, W-S., Welsh, C.A, Barres, B.A. \& Stevens, B. Nat. Neurosci. 18, 1539-1545 (2015). doi: 10.1038/nn.4142.

11. Pouille, F., Marin-Burgin, A., Adesnik, H., Atallah, B.V. \& Scanziani, M. Nat. Neurosci. 12, 1577-1585 (2009). doi: 10.1038/nn.2441.

12. Halassa, M.M. et al. Neuron 61, 213-219 (2009) doi: 10.1016/j.neuron.2008.11.024.

13. Cui, Q-Q., et al. EMBO Rep. 21, e47857 (2020) doi: 10.15252/embr.201947857

14. Di Angelatonio S., et al. Front. Cell. Neurosci. 9, 409 (2015). doi:

10.3389/fncel.2015.00409. 
Figure 1 | Inhibition of active neurons by microglial cells. a A generic neural circuit, centred on a principle neuron (PN). The PN, and an excitatory input to the circuit, both release the excitatory neurotransmitter molecule glutamate (Glu). Interneurons release the inhibitory neurotransmitter GABA. Astrocyte-derived neurotransmitters fine-tune neuronal circuits. The circuit is also inhibited by the molecule adenosine (ADO), which Badimon et al. ${ }^{2}$ show is generated in part by microglia. The negative-feedback control in the circuit maintains healthy levels of neuronal network activity. $\mathbf{b}$ When the input to the circuit is increased, GABAergic inhibition decreases the output on a rapid time scale (purple curve), while microglia-derived ADO adds a further slower component to the inhibition (green curve). c The mechanism by which microglia exert their effect involves the molecule ATP, which is released by active neurons and their associated astrocytes, and is converted into ADP by the microglial enzyme CD39. ADP acts on $\mathrm{P}_{2} \mathrm{Y}_{12}$ receptor proteins to attract microglial processes to synapses that are repeatedly active (not shown). CD39 also converts ADP into $A M P$, which is converted into adenosine (ADO) - this step might be catalysed by the enzyme CD73 on oligodendrocyte-lineage cells, neurons and perhaps microglia, and/or by tissue nonspecific alkaline phosphatase (TNAP) on astrocytes (uncertainty indicated by dashed box). ADO suppresses neuronal activity by acting on its A1 receptors. These lower the concentration of cyclic AMP which In presynaptic neurons-decreases Glu release and in postsynaptic neurons decreases the response to $\mathrm{Glu}^{8,14}$. In addition, A1 receptors activate potassium ion channels ${ }^{8}$ (so reducing neuronal excitability). 

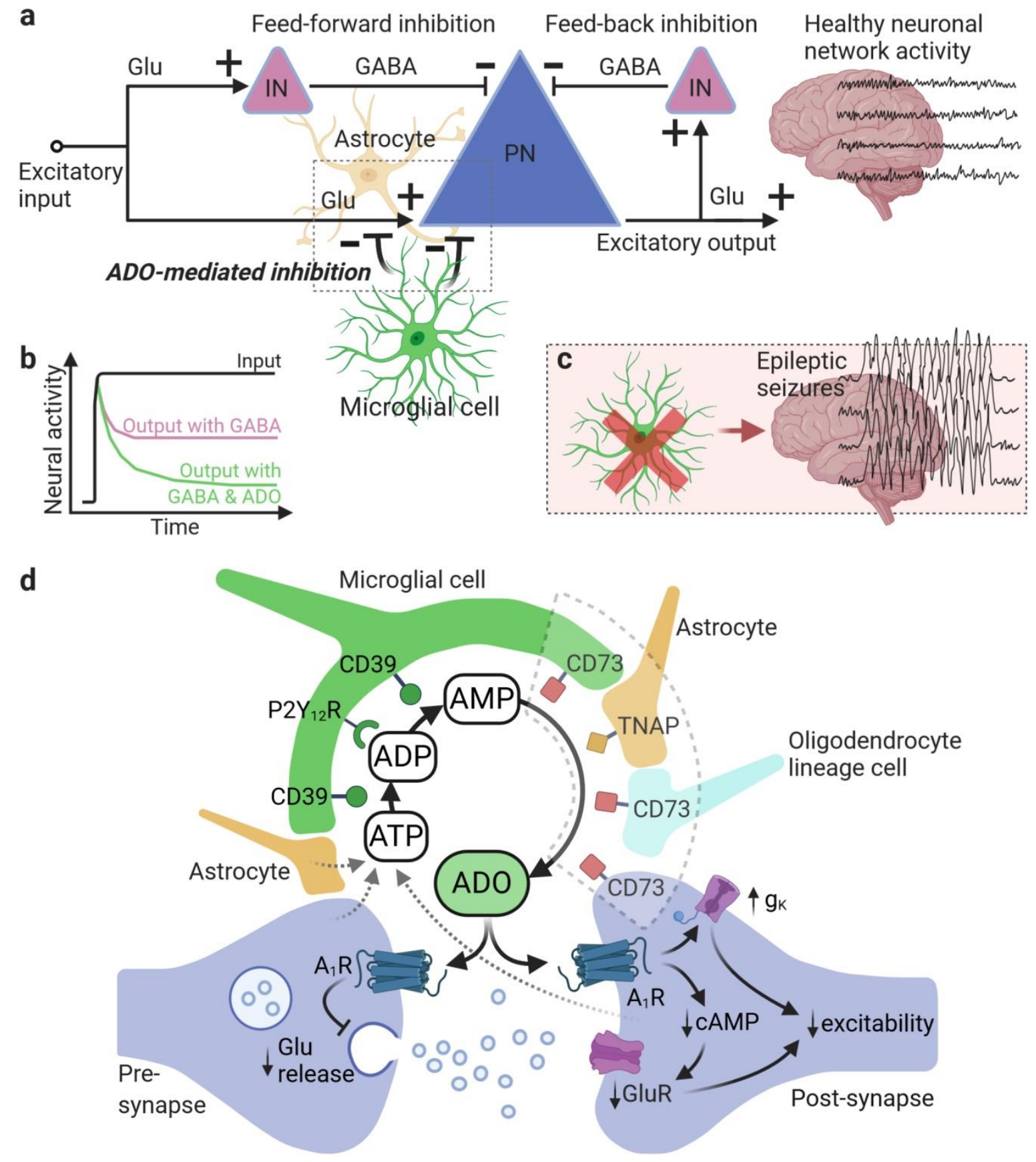

Thomas Pfeiffer and David Attwell are in the Department of Neuroscience, Physiology \& Pharmacology, University College London, Gower Street, London, WC1E 6BT, UK. E-mail: d.attwell@ucl.ac.uk 